\title{
Decreased cortical muscarinic $M 1$ receptors in schizophrenia are associated with changes in gene promoter methylation, mRNA and gene targeting microRNA
}

\author{
E Scarr ${ }^{1,10}$, JM Craig ${ }^{2,3,10}$, MJ Cairns ${ }^{4,5,10}$, MS Seo ${ }^{1,6}$, JC Galati $^{7,8}$, NJ Beveridge ${ }^{4,5}$, A Gibbons $^{1,6}$, S Juzva ${ }^{6}$, B Weinrich ${ }^{2}$, \\ M Parkinson-Bates ${ }^{9}$, AP Carroll ${ }^{4,5}$, R Saffery ${ }^{9,3}$ and B Dean ${ }^{1,6}$
}

Many studies have shown decreased cortical muscarinic M1 receptors (CHRM1) in schizophrenia (Sz), with one study showing Sz can be separated into two populations based on a marked loss of CHRM1 $(\sim 75 \%)$ in $\sim 25 \%$ of people (Def-Sz) with the disorder. To better understand the mechanism contributing to the loss of CHRM1 in Def-Sz, we measured specific markers of gene expression in the cortex of people with $\mathrm{Sz}$ as a whole, people differentiated into Def-Sz and people with $\mathrm{Sz}$ that do not have a deficit in cortical CHRM1 (Non-Def-Sz) and health controls. We now report that cortical CHRM1 gene promoter methylation and CHRM1 mRNA are decrease in Sz, Def-Sz and Non-Def-Sz but levels of the micro RNA (miR)-107, a CHRM1 targeting miR, are increased only in Def-Sz. We also report in vitro data strongly supporting the notion that miR-107 levels regulate CHRM1 expression. These data suggest there is a reversal of the expected inverse relationship between gene promoter methylation and CHRM1 mRNA in people with Sz and that a breakdown in gene promoter methylation control of CHRM1 expression is contributing to the global pathophysiology of the syndrome. In addition, our data argues that increased levels of at least one miR, miR-107, is contributing to the marked loss of cortical CHRM1 in Def-Sz and this may be a differentiating pathophysiology. These latter data continue to support the hypothesis that microRNAs (miRNA) have a role in the underlying neurobiology of Sz but argue they are differentially affected in subsets of people within that syndrome.

Translational Psychiatry (2013) 3, e230; doi:10.1038/tp.2013.3; published online 19 February 2013

\section{Introduction}

It has been argued that being able to dissect the syndrome of schizophrenia $(\mathrm{Sz})$ into its component disorders would be a significant advance towards understand the aetiologies of what should be more homogeneous component disorders. ${ }^{1}$ Significantly, a growing body of data from genetic, neuroimaging and postmortem central nervous system (CNS) studies suggest that cortical muscarinic receptors (CHRMs) are involved in the pathophysiology of $\mathrm{Sz}^{2}$ More recently, we have identified a subgroup of people with $\mathrm{Sz}(\sim 25 \%)$ that can be separated into a discrete population because they have had a marked loss ( $75 \%$ ) of cortical CHRM (Def-Sz). ${ }^{3}$ Thus, for the first time, it has become possible to study Sz as a whole and then to subsequently interrogate data divided into those with Def-Sz and those people with the disorder who do not have the deficits in CHRM (Non-Def-Sz).
In perusing the discovery of Def-Sz it is important to acknowledge that the radioligand binding assay used to delineate the two groups of people with $\mathrm{Sz}$ is highly selective for the CHRM1. ${ }^{4,5}$ In addition, it has been reported that $\mathrm{CHRM}_{1}{ }^{6}$ but not $\mathrm{CHRM} 2, \mathrm{CHRM} 3$ or $\mathrm{CHRM} 4,{ }^{6,7}$ protein is decreased in the cortex of people with the disorder. From these data we hypothesised that people with Def-Sz have a marked loss of steady-state cortical CHRM1 and that such a loss of receptor must reflect a change in either gene expression or protein degradation. To begin to address this issue, we decided to determine if critical markers of gene expression (CHRM1 gene promoter methylation, CHRM1 mRNA, CHRM1 targeting microRNA (miRNA)) were altered in the cortex of people with Sz compared with age/sex matched controls and whether these changes were more marked in Def-Sz.

\footnotetext{
${ }^{1}$ The Department of Psychiatry, The University of Melbourne, Parkville, Victoria, Australia; ${ }^{2}$ The Early Life Epigenetic Group, The Murdoch's Children's Research Institute, The Royal Children's Hospital, Parkville, Victoria, Australia; ${ }^{3}$ The Department of Paediatrics, The University of Melbourne, Parkville, Victoria, Australia; ${ }^{4}$ The School of Biomedical Sciences and Pharmacy, and Hunter Medical Research Institute, University of Newcastle, Callaghan, New South Wales, Australia; ${ }^{5}$ The Schizophrenia Research Institute, University of Sydney, Sydney, New South Wales, Australia; ${ }^{6}$ The Molecular Psychiatry Laboratory, The Florey Institute of Neuroscience and Mental Health, Parkville, Victoria, Australia; ${ }^{7}$ The Murdoch Children's Research Institute, Royal Children's Hospital, Parkville, Victoria, Australia; ${ }^{8}$ Department of Mathematics and Statistics, La Trobe University, Bundoora, Victoria, Australia and ${ }^{9}$ The Cancer and the Developmental Epigenetics Group, The Murdoch Children's Research Institute, Royal Children's Hospital, Parkville, Victoria, Australia

Correspondence: Professor B Dean, Molecular Psychiatry Laboratory, The Mental Health Research Institute, The Kenneth Myer Building, The University of Melbourne, Genetics Lane, Parkville, Victoria 3010, Australia.

E-mail: anddali@unimelb.edu.au

${ }^{10}$ These authors contributed equally to this work.

Keywords: cortex; CHRM1; muscarinic M1 receptor; postmortem CNS; schizophrenia

Received 29 November 2012; accepted 2 Decemeber 2012
} 


\section{Materials and methods}

Postmortem tissue collection and appraisal. Approval for this study was obtained from the Ethics Committee of the Victorian Institute of Forensic Medicine and the Mental Health Research and Ethics Committee of Melbourne Health. All tissue was collected following permission from the next of kin and, where a psychiatric history was identified, an extensive case history review was conducted using a structured diagnostic instrument (Diagnostic Instrument for Brain Studies ${ }^{8}$ ) allowing a consensus diagnosis according to DSM-IV criteria. ${ }^{9}$ Comprehensive medication histories were obtained, the most recently prescribed doses of antipsychotic drugs were converted to a standardized drug dose, ${ }^{10}$ those of anticholinergic drugs converted to benztropine equivalents and benzodiazepine doses converted to diazepam equivalents (Table 1). Duration of illness (time from first hospital admission to death) was also calculated.

Cadavers were refrigerated within $5 \mathrm{~h}$ of being found and CNS tissue was rapidly processed according to a standardised protocol, ${ }^{11}$ ensuring all CNS tissue was frozen to $-70{ }^{\circ} \mathrm{C}$ within $30 \mathrm{~min}$ of being removed at autopsy. Consistent refrigeration of cadavers within $5 \mathrm{~h}$ was important because data suggests such rapid refrigeration would significantly inhibit autolysis. ${ }^{12}$ To determine the quality of tissue preservation, the $\mathrm{pH}$ of CNS tissue was determined as described previously. ${ }^{13}$ For witnessed deaths, postmortem interval (PMI) was the time between death and autopsy but when death was not witnessed PMI was taken as the interval mid-way between the donor last being seen alive and being found dead.

Tissue processing. For all components of this study tissue was taken from Brodmann's Area 9 (BA 9), which was defined as the lateral surface of the frontal lobe, including the middle frontal gyrus superior to the inferior frontal sulcus, of the left CNS hemisphere.

CHRM1 gene methylation and mRNA levels. Measurement of DNA methylation was completed using tissue from 132 donors; 69 people meeting the DSM-IV criteria for the diagnoses of $\mathrm{Sz}$ and 63 people with no history of psychiatric illness (controls) (Table 1, cohort A), these cases were all included in our previous study that identified a subset of subjects with Def-Sz. ${ }^{3}$ The 69 people with Sz consisted of 49 people with $\mathrm{Sz}$ who did not have a deficit in $\left[{ }^{3} \mathrm{H}\right]$ pirenzepine binding (Non-Def-Sz) and 20 people with Sz who had Def-Sz. ${ }^{3}$

Genomic DNA extraction and bisulphite conversion. To measure CHRM1 promoter methylation, BA 9 was homogenised in DNA extraction buffer $(100 \mathrm{~mm} \mathrm{NaCl}, 10 \mathrm{~mm}$ Tris$\mathrm{HCl}$ ( $\mathrm{pH}$ 8.0), $25 \mathrm{~mm}$ EDTA, 0.5\% SDS), containing $200 \mu \mathrm{g} \mathrm{ml}^{-1}$ Proteinase $\mathrm{K}$ and incubated for $3 \mathrm{~h}$ after which DNA was isolated using a standard phenol: chloroform method. The DNA was precipitated with absolute ethanol containing $10 \%$ sodium acetate $(3 \mathrm{M}, \mathrm{pH} 5.2)$ and the DNA pellets were washed with $70 \%$ ethanol and resuspended in $100 \mu \mathrm{l}$ TE buffer (10 mm Tris-HCl (pH7.5), 1 mm EDTA). DNA was quantitated and its quality assessed spectroscopically using a Nanodrop (ThermoFisher Scientific, Walthan, MA, USA).

For each case, $2 \mu \mathrm{g}$ of genomic DNA was bisulphite converted using the MethylEasyXceed Rapid bisulphite modification kit (Human Genetic Signatures, Sydney, NSW, Australia) following the manufacturer's instructions. Using this approach sodium bisulphite selectively converts unmethylated cytosine residues to uracil nucleotides, leaving methylated cytosine unchanged. All samples were processed in parallel to ensure consistency.

PCR amplification. Primers for CHRM1 were designed using PrimerExpress 3.0 following sequence bisulphite conversion using Methyl Primer Express v1.0 software. (Supplementary Table 1) for primer sequences. Three amplicons were designed, subsequent to a bioinformatic analysis, to characterise DNA methylation level and distribution at the CHRM1 gene promoter (See Supplementary Figure 1). To reduce technical variability, three replicate amplifications were performed in $15 \mu$ reactions containing $10 \mathrm{ng}$ bisulphite-converted DNA, 1X FastStart PCR master mix (Roche Diagnostics Australia Pty Castle Hill, NSW, Australia) and $3 \mathrm{~nm}$ each of forward and reverse primers. The amplification conditions were: initial denaturation at $95^{\circ} \mathrm{C}$ for $2 \mathrm{~min}$, followed by five cycles of $95^{\circ} \mathrm{C}$ for $30 \mathrm{~s}$, $56{ }^{\circ} \mathrm{C}$ for $30 \mathrm{~s}, 72^{\circ} \mathrm{C}$ for $90 \mathrm{~s}$, then 35 cycles of $95^{\circ} \mathrm{C}$ for $30 \mathrm{~s}, 60^{\circ} \mathrm{C}$ for $30 \mathrm{~s}, 72^{\circ} \mathrm{C}$ for $90 \mathrm{~s}$, and a final extension at $72{ }^{\circ} \mathrm{C}$ for $7 \mathrm{~min}$.

DNA methylation. DNA methylation levels were determined as described previously ${ }^{14}$ using MassARRAY EpiTYPER (Sequenom, QLD, San Diego, CA, USA), based on MALDITOF mass spectrometry. In short, bisulphite converted DNA was amplified with primers containing a T7-promoter tag and the amplification products cleaned with shrimp alkaline phosphatase (SAP, Sequenom, San Diego, CA, USA). After purification, in vitro transcription and $T$ specific cleavage was performed. Each reaction was then spotted onto a Maldimatrix-containing SpectroCHIPS (Sequenom) and subjected to MALDI-TOF MS. The mass spectra was collected by MassARRAY Spectrometer and analysed by EpiTYPER v.1.0 software (Sequenom). Data was obtained for each CpG-containing fragment (unit), with most units containing single CpGs, and two units (0304_CpG5_6 and 06_CpG3_4) containing two CpGs. For simplicity, all $\mathrm{CpG}$ units are referred to as 'sites' in the Results.

The Sequenom technology, like all sequencing technologies, ${ }^{15}$ gives results on single nucleotides that is of varying certainty and there are issues relating to the difficulties in measuring the status of every potential nucleotide methylation status. ${ }^{16}$ Therefore to ensure our data analyses only included high quality data we applied a stringent quality control process to remove potentially unreliable measurements before analysis. ${ }^{14}$ Firstly, all data from CpG-containing fragments flagged by EpiTYPER as having low or high mass (outside the MS analytical window), or overlapping MS peaks were discarded. Secondly, CpG analytic units that failed to produce data for $>30 \%$ of samples were also discarded (unreliable CpG unit), and samples with more than $30 \%$ missing data points within an amplicon (unreliable sample) had all methylation values for 
Table 1 Donor demographics, treatment and CNS collection data for cases from which tissue was collected from BA 9 for this study

\begin{tabular}{|c|c|c|c|c|c|c|c|c|c|c|c|c|}
\hline $\begin{array}{l}\text { Cohort } \\
\text { groups }\end{array}$ & Diagnoses & $\mathrm{n}$ & $\begin{array}{c}\text { Sex } \\
(M / F)\end{array}$ & $\begin{array}{c}\text { Sui } \\
(Y / N)\end{array}$ & $\begin{array}{c}\text { Age } \\
\text { (years) }\end{array}$ & $p H$ & $\begin{array}{c}\text { PMI } \\
\text { (hour) }\end{array}$ & $\begin{array}{c}\text { Brain } \\
\text { weight } \\
\text { (gms) }\end{array}$ & $\begin{array}{c}D O I \\
\text { (years) }\end{array}$ & $A P D$ & $A C D$ & $D Z D$ \\
\hline \multirow[t]{4}{*}{ A } & Control & 63 & $47 / 16$ & & $43 \pm 2$ & $6.33 \pm 0.02$ & $41 \pm 1.8$ & $1418 \pm 19$ & & & & \\
\hline & $\mathrm{Sz}$ & 69 & $51 / 18$ & & $44 \pm 2$ & $6.31 \pm 0.02$ & $40 \pm 1.5$ & $1427 \pm 20$ & $18 \pm 2$ & $624 \pm 75$ & $1.7 \pm 0.12$ & $13 \pm 2.8$ \\
\hline & Def-Sz & 20 & $16 / 4$ & $7 / 13$ & $46 \pm 4$ & $6.25 \pm 0.04$ & $39 \pm 2.7$ & $1461 \pm 45$ & $20 \pm 4$ & $593 \pm 124$ & $1.8 \pm 0.09$ & $3.5 \pm 0.6$ \\
\hline & Non-Def-Sz & 49 & $35 / 14$ & $26 / 23$ & $46 \pm 2$ & $6.12 \pm 0.05$ & $42 \pm 2.0$ & $1413 \pm 23$ & $18 \pm 2$ & $638 \pm 94$ & $1.5 \pm 0.18$ & $19 \pm 4.0$ \\
\hline \multirow[t]{4}{*}{ B } & Control & 15 & $12 / 3$ & & $45 \pm 4$ & $6.30 \pm 0.05$ & $39 \pm 3.8$ & $1355 \pm 38$ & & & & \\
\hline & $\mathrm{Sz}$ & 27 & $21 / 6$ & & $45 \pm 3$ & $6.19 \pm 0.05$ & $40 \pm 2.8$ & $1393 \pm 34$ & $18 \pm 3$ & $602 \pm 109$ & $1.8 \pm 0.10$ & $5.7 \pm 1.8$ \\
\hline & Def-Sz & 14 & $11 / 3$ & $5 / 9$ & $44 \pm 5$ & $6.25 \pm 0.06$ & $40 \pm 3.5$ & $1425 \pm 55$ & $18 \pm 4$ & $623 \pm 147$ & $1.8 \pm 0.12$ & $3.1 \pm 0.6$ \\
\hline & Non-Def Sz & 13 & $10 / 3$ & $3 / 10$ & $46 \pm 5$ & $6.13 \pm 0.08$ & $40 \pm 4.5$ & $1355 \pm 36$ & $18 \pm 4$ & $568 \pm 169$ & $1.7 \pm 0.20$ & $12 \pm 4.4$ \\
\hline
\end{tabular}

Cohort definitions: Cohort Group A: used for the determination of CHRM1 mRNA and methylation of the promoter region.

Cohort Group B: a subgroup selected at random from cohort A used for studies on miRNAs.

Abbreviations: ACD, last recorded anticholinergic drug doses in benztropine equivalents; APD, last recorded antipsychotic drug doses in chlorpromazine equivalent; Def-Sz, $\left[{ }^{3} \mathrm{H}\right]$ pirenzepine binding deficient schizophrenia; DOI, duration of illness; DZD, last recorded benzodiazepine doses in diazepam equivalents; $\mathrm{F}$, female; $\mathrm{M}$, male; Non-Def-Sz, subjects with schizophrenia without deficits in $\left[{ }^{3} \mathrm{H}\right]$ pirenzepine binding; N, no; PMI, postmortem interval; Sui, suicide; Sz, schizophrenia; Y, yes.

that sample set to 'missing'. Finally, technical replicates showing $\geqslant 5 \%$ absolute difference from the median value of a set of technical replicates were set to 'missing' and only samples with at least two successful technical replicates were analysed.

\section{Quantifying CHRM1 mRNA}

In situ hybridisation. In situ hybridisation was performed as described previously. ${ }^{17}$ Briefly, $6 \times 10 \mu \mathrm{m}$ sections were cut from the BA 9 of each subject, fixed in $4 \%$ paraformaldehyde, placed in fresh $0.25 \%$ acetic anhydride in $0.1 \mathrm{~m}$ triethanolamine $\mathrm{HCl}(\mathrm{pH} 8.0)$, dehydrated, delipidated and equilibrated in $95 \%$ ethanol before being dried.

To increase specificity, the oligonucleotide probe contained a mix of three antisense sequences, the probes were complementary to bases $4-51,721-768$ and $811-853$ of human CHRM1 mRNA (accession no.: X52068). ${ }^{6}$ The oligonucleotides were labelled with $\left[{ }^{35}\right.$ S $]$ ATP using the NEN Oligonucleotide $3^{\prime}$ end labelling system and purified using a NENSORB 20 purification cartridge (Perkin Elmer, Glen Waveryly, VIC, Australia).

For hybridisation, the probe was prepared at $1 \times 10^{6} \mathrm{dpm}$ of $\left[{ }^{35} \mathrm{~S}\right]$ ATP per $50 \mu \mathrm{l}$ of hybridisation buffer (formamide $(50 \%)$, $\mathrm{NaCl}(600 \mathrm{~mm})$, Tris- $\mathrm{HCl}(80 \mathrm{~mm})$, ethylenediamine tetraacetate $(4 \mathrm{~mm})$, sodium pyrophosphate $(0.1 \%)$, SDS $(0.2 \%)$, sodium heparin $\left(2 \mathrm{mg} \mathrm{ml}^{-1}\right)$, dextran sulphate $(10 \%)$ and dithiothreitol $(100 \mathrm{~mm})$ ) and incubated with tissue sections for $20 \mathrm{~h}$ at $37^{\circ} \mathrm{C}$, in a humidified chamber. Two sections were incubated with the radioactive oligonucleotide probe (total binding) while two sections were incubated with radioactive probes in the presence of excess non-radioactive probes (non-specific binding). Hybridisation to mRNA was confirmed by incubating two sections, preincubated in $0.02 \%$ RNaseA, with the radioactive probe (negative control).

After hybridisation, all sections were washed ${ }^{18}$ and dried before being apposed to BAS-SR2025 imaging plates (Fujifilm, Tokyo, Japan), with $\left[{ }^{14} \mathrm{C}\right]$ microscales (GE Healthcare, Rydalmere, NSW, Australia) for 4 weeks before being scanned in the BAS 5000 high resolution phosphoimager (Fujifilm). ${ }^{19}$ The signal intensity of the phosphoimages were measured against those on the $\left[{ }^{14} \mathrm{C}\right]$ microscales using AIS image analysis software. Results were expressed as TB minus NSB in $\mathrm{dpm} \times 10^{3}$ per mg estimated wet weight tissue equivalents.

miRNA. This component of the study used Brodmann's Area 9 from 14 people with Def-Sz, 13 people with Non-Def Sz and 15 control people (Table 1B).

CHRM1 miRNA target site analysis. CHRM1 target site prediction was accomplished using the miRNA target database miRGen (http://diana.pcbi.upenn.edu/cgi-bin/miRGen/v3/Targets.cgi) (Supplementary Table 2). This service provides meta-analysis of established miRNA target prediction algorithms that search the 3 UTR sequences for homology and putative affinity with known miRNA sequences. ${ }^{20}$ These algorithms also have weightings that enable the appreciation of conservation between 3 UTR motifs in vertebrate species that are more likely to have functional significance. An important feature of the miRGen database is that it provides output on the amalgamation of predictions from multiple algorithms to yield a broad low stringency appraisal of a given target gene or more stringent outputs that consider the intersection of miRNA target sites that satisfy multiple algorithms including miRanda, PictaR, TargetScanS and DIANA-microT.

RNA purification and first-strand complementary DNA synthesis. Total RNA was isolated from $100 \mathrm{mg}$ frozen cortical samples with $1.0 \mathrm{ml}$ TRIzol reagent (Invitrogen, Mulgrave, VIC, Australia) for human tissue and QIAGEN (Doncaster, VIC, Australia) RNA extraction kit for mouse tissue, according to the manufacturer's instructions. Following Trizol extraction, the RNA was treated with $0.1 \mathrm{U} \mu \mathrm{l}^{-1}$ DNAse in $1 \times$ DNAse buffer (Invitrogen, Australia) to remove genomic DNA contamination, extracted using phenol $(\mathrm{pH}$ 4.2): chloroform extraction and ethanol precipitated. complementary DNA was synthesised as described previously. ${ }^{21}$

miRNA expression. miRNA expression analysis was performed using miRNA specific relative quantitative real-time PCR as described previously. ${ }^{22}$ Primer sequences used in reverse transcription reaction and quantitative real-time PCR 
are provided in Supplementary Table 3. Relative expression was determined with respect to the geometric means of three constitutively expressed small nuclear RNA (U6, U44 and U49) by subtracting the geometric means of their cycle threshold value from the cycle threshold value derived for the miRNA. ${ }^{23}$ All reactions were subjected to post amplification dissociation curve analysis to provide guidance on reaction specificity. Initial confirmation of amplicon length was also accomplished through gel electrophoresis.

Reporter gene assay. Luciferase reporter gene analysis of miRNA function was conducted as described previously. ${ }^{24}$ Briefly, HEK-293 cell cultures were seeded into 24-well plates and maintained in DMEM with $10 \%$ (vol/vol) foetal calf serum, $20 \mathrm{~mm}$ HEPES, $0.15 \%$ (wt/vol) sodium bicarbonate and $2 \mathrm{~mm}$ L-glutamine at $37{ }^{\circ} \mathrm{C}$ with $5 \% \mathrm{CO} 2$ and $90 \%$ humidity. Transfections were performed $24 \mathrm{~h}$ postseeding with recombinant $\mathrm{CHRM1-Firefly} \mathrm{luciferase} \mathrm{reporter} \mathrm{gene}$ constructs, $100 \mathrm{~nm}$ synthetic miRNA or LNA modified antimiR oligonucleotide in Lipofectamine 2000 according to manufacturer's instructions (Invitrogen). Reporter gene constructs containing 3 UTR sequences encoding target gene miRNA recognition elements were produced through the ligation of a synthetic double stranded cassette compatible with Spel and Hind III digested PMIR-REPORT vector (Invitrogen) (see Supplementary Figure 2). Reporter gene silencing in response to miRNA cotransfection was monitored with respect to a control plasmid expressing Renilla luciferase ( $p R L-T K$ ) using the dual luciferase reporter assay (Promega, Alexandria, NSW, Australia). Non-specific effects associated with transfection were controlled by comparison to cells cotransfected with mutant miRNAs or mutant anti-miRs.

$\left[{ }^{3} \mathrm{H}\right]$ pirenzepine binding. The data on $\left[{ }^{3} \mathrm{H}\right]$ pirenzepine binding used in this study was originally generated in an earlier study. ${ }^{3}$ The data pertaining to the relevant cohorts was collated and then reanalysed within the parameters of each portion of this study.

Statistics. The distribution of all data was determined using the D'Agostino and Pearson omnibus normality test as this is best for determining data distribution in small cohorts. ${ }^{25}$ Demographic, pharmacological and CNS collection data were compared using a student's $t$-test at the levels of $\mathrm{Sz}$ and a one-way analysis of variance when comparing Def-Sz and Non-Def-Sz to controls. Where data were found to be mixed between parametric and non-parametric distributions the General Linear Model was used to identify significant variance whereas Mann-Whitney U or Kruskal-Wallis tests were used when all data to be analysed was shown to have a non-parametric distribution. Gender frequency across diagnostic cohorts was assessed using the $\chi^{2}$ test. Potential biological relationships and confounding factors were integrated using linear regression analysis. Due to small cohort sizes only strong relationships $\left(r^{2}=0.49\right)$ where the regression deviated significantly were considered as being of interest. ${ }^{26}$

Most analyses were conducted using Prism 5.01 (Graphpad Software, La Jolla, CA, USA) but the General Linear Model was computed using Minitab 15 (Minitab, State
College, PA, USA) while the methylation data was analysed using Stata 11 (StataCorp., 2010; College Station, TX, USA).

\section{Results and discussion}

\section{Demographics, pharmacological history and CNS collection data}

mRNA and methylation cohorts:. The cases used in this component of the study were part of the cohorts used in the study that identified Def-Sz ${ }^{27}$ with the cohort sizes being limited by availability of tissue from BA 9. There were no significant differences in mean age $(P=0.63 ; P=0.92)$, PMI $(P=0.75 ; P=0.84)$, CNS pH $(P=0.19 ; P=0.10)$, CNS weight $(P=0.54, P=0.23)$ or gender frequency $(P=1.00$; $P=0.11)$ when comparing Sz to control or Def-Sz, Non-DefSz and controls, respectively, for the larger cohorts (Table 1: Cohort Groups A). In comparing Def-Sz and Non-Def-Sz there were no significant differences in mean duration of illness $(P=0.87)$, the last recorded dose of antipsychotic $(P=0.73)$ or anticholinergic $(P=0.15)$ drugs but the Def-Sz had lower final recorded doses of benzodiazepines $(P=0.01)$. The frequency of benzodiazepine prescription did not differ between Def-Sz and Non-Def-Sz $(P=0.60)$. This would suggest that the symptoms of schizophrenia targeted by benzodiazepines (distress, insomnia and behavioural disturbances secondary to psychosis ${ }^{28}$ ) are more responsive to benzodiazepine treatment in subjects with DefSz by a mechanism that has yet to be understood.

miRNA cohort:. The cohorts for this study were selected based on diagnoses, whether or not they were shown to be Def-Sz or Non-Def-Sz by measuring $\left[{ }^{3} \mathrm{H}\right]$ pirenzepine binding, the availability of tissue from BA 9 and limitations in sample processing capacity of the technique. Together these factors contributed to resulted in smaller cohorts being studied when measuring miRNA. There were no significant differences in mean age $(P=0.55 ; P=0.83)$, $\mathrm{PMI}(P=0.71$; $P=0.93)$, CNS pH $(P=0.11 ; P=0.16)$, CNS weight $(P=0.44 ; P=0.29)$ or gender frequency $(P=1.00 ; P=0.99)$ when comparing $\mathrm{Sz}$ to control or Def-Sz, Non-Def-Sz and controls, respectively (Table 1: Cohort Groups B). In comparing Def-Sz and Non-Def-Sz there were no significant differences in mean duration of illness $(P=0.96)$, the last recorded dose of antipsychotic drugs $(P=0.59)$ or anticholinergic drugs $(P=0.50)$ but the Def-Sz had lower final recorded doses of benzodiazepines $(P=0.04)$.

CHRM1 Gene methylation. Ten potential CpG sites were identified across the putative CHRM1 promoter region (Supplementary Figure 1). There was a significant reduction in levels of methylation in four of these sites in people with $\mathrm{Sz}$ (Table 2) with further analyses revealing a significant reduction in levels of DNA methylation in three sites in people with Def-Sz and Non-Def-Sz (Table 3), plus an addition site in the Non-Def-Sz. Examining the pooled methylation data showed that there was a significant decrease in methylation associated with the diagnoses of $\mathrm{Sz}$ but not with other factors such as suicide, gender, age, tissue $\mathrm{pH}$ or $\mathrm{PMI}$ (Table 4). In addition, there were very similar reductions in DNA methylation in all CpG sites in 
Table 2 Difference in mean CHRM1 DNA methylation at individual CpG units for schizophrenia vs control groups (after adjustment for death by suicide, sex, age, pH and $\mathrm{PMI})$

\begin{tabular}{|c|c|c|c|c|c|c|c|c|c|}
\hline \multirow[t]{2}{*}{ CpG unit } & \multirow[t]{2}{*}{ Genome coordinate } & \multicolumn{2}{|c|}{ Number } & \multicolumn{2}{|c|}{ Methylation } & \multicolumn{4}{|c|}{ Difference (Sz minus Controls) } \\
\hline & & $S z$ & Control & $S z$ & Control & $\triangle$ Mean Methylation & $\mathrm{P}$ & 95 & $C l$ \\
\hline chrm1_0401_CpG2 & 62445428 & 56 & 54 & $16.0 \%$ & $17.9 \%$ & $-2.9 \%$ & 0.17 & $-7.0 \%$ & $1.2 \%$ \\
\hline chrm1_0401_CpG3 & 62445440 & 64 & 60 & $7.3 \%$ & $7.4 \%$ & $-0.4 \%$ & 0.55 & $-1.8 \%$ & $1.0 \%$ \\
\hline chrm1_0304_CpG2 & 62445521 & 55 & 49 & $15.8 \%$ & $17.0 \%$ & $-2.2 \%$ & 0.072 & $-4.5 \%$ & $0.2 \%$ \\
\hline chrm1_0304_CpG4 & 62445617 & 48 & 44 & $25.6 \%$ & $28.0 \%$ & $-5.3 \%$ & 0.006 & $-9.1 \%$ & $-1.5 \%$ \\
\hline chrm1_0304_CpG5_6 & 62445641 & 54 & 52 & $18.7 \%$ & $20.8 \%$ & $-3.2 \%$ & 0.017 & $-5.9 \%$ & $-0.6 \%$ \\
\hline chrm1_06_CpG2 & 62445645 & 58 & 55 & $15.1 \%$ & $16.4 \%$ & $-2.7 \%$ & 0.010 & $-4.8 \%$ & $-0.6 \%$ \\
\hline chrm1_06_CpG3_4 & 62445657 & 63 & 60 & $15.2 \%$ & $15.7 \%$ & $-0.9 \%$ & 0.32 & $-2.5 \%$ & $0.8 \%$ \\
\hline chrm1_06_CpG7 & 62445700 & 61 & 56 & $26.7 \%$ & $26.8 \%$ & $-1.1 \%$ & 0.34 & $-3.4 \%$ & $1.2 \%$ \\
\hline chrm1_06_CpG8 & 62445953 & 58 & 57 & $32.8 \%$ & $34.5 \%$ & $-2.4 \%$ & 0.046 & $-4.9 \%$ & $0.0 \%$ \\
\hline chrm1_06_CpG9 & 62445992 & 59 & 55 & $41.7 \%$ & $44.1 \%$ & $-2.6 \%$ & 0.075 & $-5.4 \%$ & $0.3 \%$ \\
\hline
\end{tabular}

Abbreviations: $\mathrm{Cl}$, confidence interval; Sz, schizophrenia.

Bold entries indicate $P<0.05$.

Table 3 Difference in mean methylation for Def and Non-Def schizophrenia groups minus controls at individual CpG units (after adjustment for sex, age pH, PMI and death by suicide)

\begin{tabular}{|c|c|c|c|c|c|c|c|c|c|c|c|c|}
\hline \multirow[t]{3}{*}{ CpG unit } & \multirow{3}{*}{$\begin{array}{c}\text { Genome } \\
\text { coordinate }\end{array}$} & \multicolumn{3}{|c|}{ Number } & \multicolumn{4}{|c|}{ Def-Sz vs controls } & \multicolumn{4}{|c|}{ Non-Def-Sz vs controls } \\
\hline & & \multicolumn{2}{|c|}{$S z$} & \multirow[t]{2}{*}{ Control } & \multirow{2}{*}{$\begin{array}{c}\triangle \text { Mean } \\
\text { methylation }\end{array}$} & \multirow[t]{2}{*}{$\mathrm{P}$} & \multicolumn{2}{|c|}{$95 \% \mathrm{Cl}$} & \multirow{2}{*}{$\begin{array}{c}\triangle \text { Mean } \\
\text { methylation }\end{array}$} & \multirow[t]{2}{*}{$\mathrm{P}$} & \multicolumn{2}{|c|}{$95 \% \mathrm{Cl}$} \\
\hline & & Def & $\begin{array}{l}\text { Non- } \\
\text { Def }\end{array}$ & & & & & & & & & \\
\hline chrm1_0401_CpG2 & 62445428 & 18 & 38 & 54 & $-3.9 \%$ & 0.12 & $-8.8 \%$ & $1.0 \%$ & $-2.2 \%$ & 0.34 & $-6.8 \%$ & $2.4 \%$ \\
\hline chrm1_0401_CpG3 & 62445440 & 20 & 44 & 60 & $-0.7 \%$ & 0.40 & $-2.4 \%$ & $1.0 \%$ & $-0.2 \%$ & 0.78 & $-1.8 \%$ & $1.3 \%$ \\
\hline chrm1_0304_CpG2 & 62445521 & 16 & 39 & 49 & $-2.5 \%$ & 0.08 & $-5.4 \%$ & $0.3 \%$ & $-1.9 \%$ & 0.16 & $-4.5 \%$ & $0.7 \%$ \\
\hline chrm1_0304_CpG4 & 62445617 & 14 & 34 & 44 & $-6.8 \%$ & $<0.01$ & $-11.6 \%$ & $-2.1 \%$ & $-4.4 \%$ & 0.04 & $-8.6 \%$ & $-0.3 \%$ \\
\hline chrm1_0304_CpG5_6 & 62445641 & 16 & 38 & 52 & $-3.8 \%$ & 0.02 & $-7.1 \%$ & $-0.6 \%$ & $-2.8 \%$ & 0.07 & $-5.7 \%$ & $0.2 \%$ \\
\hline chrm1_06_CpG2 & 62445645 & 17 & 41 & 55 & $-3.1 \%$ & 0.01 & $-5.5 \%$ & $-0.6 \%$ & $-2.4 \%$ & 0.04 & $-4.7 \%$ & $-0.1 \%$ \\
\hline chrm1_06_CpG3_4 & 62445657 & 20 & 43 & 60 & $-0.8 \%$ & 0.42 & $-2.9 \%$ & $1.2 \%$ & $-0.9 \%$ & 0.37 & $-2.8 \%$ & $1.0 \%$ \\
\hline chrm1_06_CpG7 & 62445700 & 20 & 41 & 56 & $-1.0 \%$ & 0.46 & $-3.7 \%$ & $1.7 \%$ & $-1.2 \%$ & 0.36 & $-3.8 \%$ & $1.4 \%$ \\
\hline chrm1_06_CpG8 & 62445953 & 17 & 41 & 57 & $-2.1 \%$ & 0.16 & $-5.0 \%$ & $0.8 \%$ & $-2.7 \%$ & 0.05 & $-5.4 \%$ & $0.0 \%$ \\
\hline chrm1_06_CpG9 & 62445992 & 20 & 39 & 55 & $-2.2 \%$ & 0.19 & $-5.5 \%$ & $1.1 \%$ & $-2.9 \%$ & 0.08 & $-6.1 \%$ & $0.3 \%$ \\
\hline
\end{tabular}

Abbreviations: $\mathrm{Cl}$, confidence interval; chrm1, cortical muscarinic M1 receptors; Def-Sz, [ $\left.{ }^{3} \mathrm{H}\right]$ pirenzepine binding deficient schizophrenia; Non-Def-Sz, subjects with schizophrenia without deficits in $\left[{ }^{3} \mathrm{H}\right]$ pirenzepine binding; $\mathrm{Sz}$, schizophrenia.

Bold entries indicate $P<0.05$.

Table 4 Predictors of CHRM1 DNA methylation

\begin{tabular}{lrrrr}
\hline \multicolumn{5}{c}{ CHRM1 DNA methylation } \\
\hline Covariate & Coefficient & $\mathrm{P}$ & $95 \% \mathrm{Cl}$ \\
\hline Schizophrenia vs controls & & & & \\
$\quad$ Schizophrenia & $-\mathbf{2 . 2} \%$ & $<\mathbf{0 . 0 1}$ & $-\mathbf{3 . 8} \%$ & $-\mathbf{0 . 6 \%}$ \\
Suicide & $1.7 \%$ & 0.09 & $-0.3 \%$ & $3.7 \%$ \\
Sex (males minus females) & $-0.5 \%$ & 0.53 & $-2.0 \%$ & $1.0 \%$ \\
Age (z-score) & $-0.4 \%$ & 0.33 & $-1.1 \%$ & $0.4 \%$ \\
pH (z-score) & $-0.4 \%$ & 0.36 & $-1.2 \%$ & $0.4 \%$ \\
Post mortem interval & $-0.1 \%$ & 0.86 & $-0.8 \%$ & $0.7 \%$ \\
(z-score) & & & & \\
Def-Sz, non-Def-Sz and controls & & & & \\
non-Def_Sz minus controls & $-\mathbf{2 . 0} \%$ & $\mathbf{0 . 0 3}$ & $-\mathbf{3 . 9} \%$ & $-\mathbf{0 . 2} \%$ \\
Def_Sz minus controls & $-\mathbf{2 . 4 \%}$ & $\mathbf{0 . 0 2}$ & $-\mathbf{4 . 5} \%$ & $-\mathbf{0 . 4 \%}$ \\
Def-Sz minus non-Def-Sz & $-0.4 \%$ & 0.70 & $-2.5 \%$ & $1.7 \%$ \\
& & & & \\
&
\end{tabular}

Abbreviations: $\mathrm{Cl}$, confidence interval; Def-Sz, $\left[{ }^{3} \mathrm{H}\right]$ pirenzepine binding deficient schizophrenia; Non-Def-Sz, subjects with schizophrenia without deficits in $\left[{ }^{3} \mathrm{H}\right]$ pirenzepine binding; Sz, schizophrenia.

Bold entries indicate $P<0.05$. tissue from both Def-Sz and Non-Def-Sz compared with controls, which meant there was no difference in DNA methylation between the two forms of Sz.

In situ hybridisation and in situ radioligand binding. Levels of oligonucleotide hybridisation to CHRM1 mRNA and $\left[{ }^{3} \mathrm{H}\right]$ pirenzepine binding were homogenous throughout the cortical laminae and therefore a single integrated measure was taken across the cortex. Levels of CHRM1 mRNA $(P=0.01)$ and $\left[{ }^{3} \mathrm{H}\right]$ pirenzepine binding $(P<0.001)$ were significantly lower in the cortex of people with $\mathrm{Sz}$ compared with controls (Figure 1a).

There was a significant variation in levels of oligonucleotide hybridisation to CHRM1 mRNA with group between people with Def-Sz, Non-Def-Sz and controls $(F=9.09$, d.f. $=2148$, $P<0.0001)$ due to lower levels of mRNA in the cortex from people with Def-Sz $(P=0.001)$ and Non-Def-Sz $(P=0.004)$ compared with controls (Figure 1b) but levels of CHRM1 mRNA in Def-Sz were not significantly different to that in 
Non-Def-Sz $(P=0.63)$. As would be predicted, there was also significant variation in levels of $\left[{ }^{3} \mathrm{H}\right]$ pirenzepine binding with group $(\mathrm{F}=95.6$, d.f. $=2148, P<0.0001)$ due to decreased levels of binding in Def-Sz $(P<0.0001)$ and Non-Def-Sz $(P=0.02)$ compared with controls.

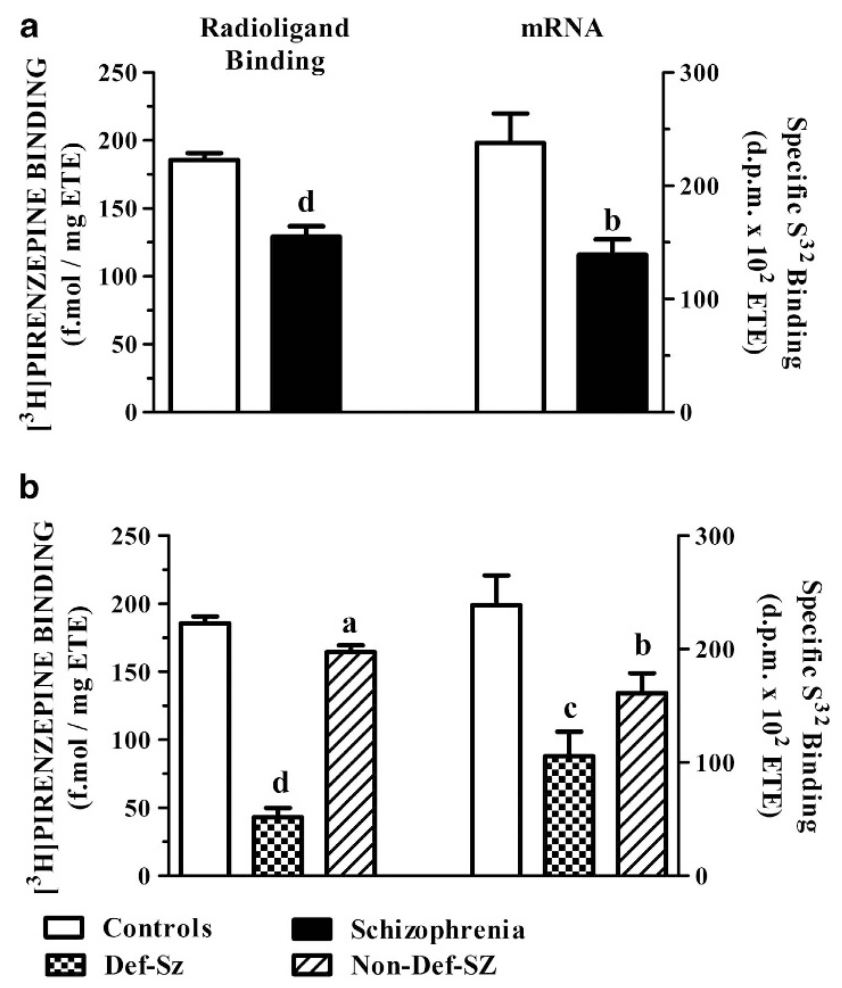

$\mathbf{a}=\mathrm{p}<0.05 ; \mathbf{b}=\mathrm{p}<0.01 ; \mathbf{c}=\mathrm{p}<0.001 ; \mathbf{d}=\mathrm{p}<0.0001$

Figure 1 Levels (Mean \pm s.e.m.) of $\left[{ }^{3} \mathrm{H}\right]$ pirenzepine binding and oligonucleotide CHRM1 probe hybridisation to Brodmann's area 9 (BA 9) from subjects with. (a): schizophrenia and controls and (b): Def-Sz, Non-Def-Sz and controls.
miRNA. Initially, to identify potential miRNA regulators of CHRM1 we performed a low stringency search of the miRGen miRNA target prediction database, using the 'union' of their algorithms. ${ }^{20}$ This suggested that CHRM1 could be influenced by up to 32 different miRNA (Supplementary Table 2). However, a more stringent appraisal of targets using the 'intersection' of different algorithms identified a single miR-107 as the miRNA most likely to target CHRM1 expression. The miR107 target site in the CHRM1 3 UTR is only one of two that display a highly conserved complete seven nucleotide seed-paring region (Supplementary Figure 2) and, additionally, has previously been shown to be increased in postmortem superior temporal cortex and dorsolateral prefrontal cortex from people with $\mathrm{Sz}^{23}$ Similar analyses suggested it was highly unlikely that miR-16 would target $C H R M 1$ expression and so levels of this $\mathrm{miR}$ were measured to determine if any change in miR-107 was due to a generalised change in levels of $\mathrm{miR}$ in the cortex of people with Sz.

In the cohorts used in the study of miRNA, levels of cortical $\left[{ }^{3} \mathrm{H}\right]$ pirenzepine binding $(P=0.0004)$ and CHRM1 mRNA $(P=0.03)$ were decreased in BA 9 from people with $\mathrm{Sz}$ (Figure 2a) but neither levels of miR-16 $(P=0.50)$ nor miR$107(P=0.19)$ varied significantly with diagnosis (Figure $2 \mathrm{~b}$ ).

In comparing data from Def-Sz, Non-Def-Sz and controls, levels of miR-107 (F=4.25, d.f. $=2,40, P=0.02)$ and $\left[{ }^{3} \mathrm{H}\right]$ pirenzepine binding $(\mathrm{F}=72.3$, d.f. $=2,40, P<0.0001)$ varied with group, levels of $C H R M 1$ mRNA showed a trend to varying with group $(\mathrm{F}=2.68$, d.f. $=2,40, P=0.08)$ whereas levels of miR-16 did not vary with group $(\mathrm{F}=0.65$, d.f. $=2,40, P=0.52)$ (Figures 2c and d). The variance in $\mathrm{miR}-107$ was due to increased levels of that miRNA in the cortex of people with Def-Sz compared with controls $(P<0.05)$ but levels of miR107 were not significantly different between the Non-Def-Sz and controls. The variation in $\left[{ }^{3} \mathrm{H}\right]$ pirenzepine binding with group was due to decreased levels of binding in cortex from people with Def-Sz $(P<0.001)$, but not Non-Def-Sz, compared with controls. The trend to variance in levels of $C H R M 1$

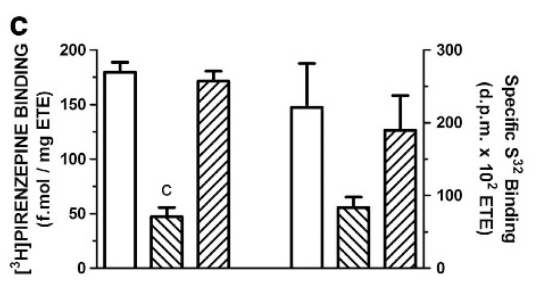

d
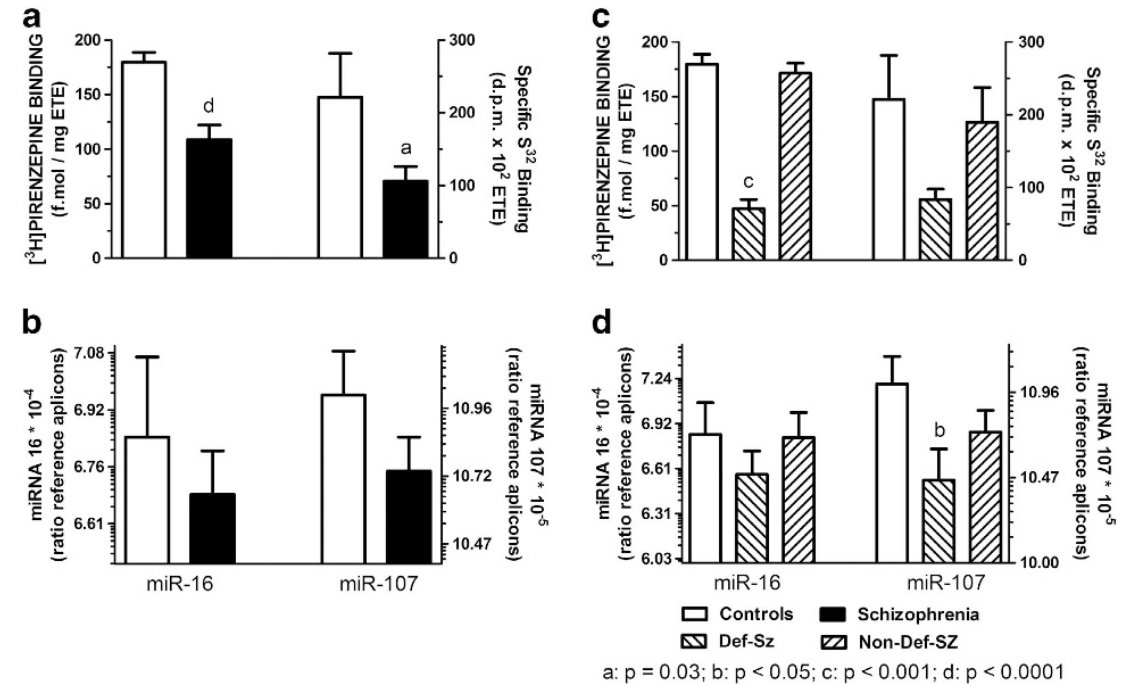

Figure 2 (a): Levels (Mean \pm s.e.m.) of $\left[{ }^{3} \mathrm{H}\right]$ pirenzepine binding and oligonucleotide CHRM1 probe hybridisation to BA 9 from the cohort of subjects with schizophrenia and control subjects used for the study of miRNA and. (b): Levels (Mean \pm s.e.m.) of miR-16 and miR-107 in the same region from the individuals. (c): Levels (Mean \pm s.e.m.) of $\left[{ }^{3} \mathrm{H}\right]$ pirenzepine binding and oligonucleotide CHRM1 probe hybridisation to BA 9 from the cohort of subjects with Def-Sz, Non-Def-Sz and control subjects used for the study of miRNA and (d): Levels (Mean \pm s.e.m.) of miR-16 and miR-107 in the same region from the individuals. 
mRNA was predominantly due to lower levels in the Def-Sz group ( $t$-test compared with controls $P=0.03$ ) rather from the Non-Def-Sz ( $t$-test compared with controls $P=0.67$ ).

Potential biological relationships. For the cohorts of people used for the study of $\left[{ }^{3} \mathrm{H}\right]$ pirenzepine binding, CHRM1 mRNA and gene promoter methylation, there were no strong correlations between levels of $\left[{ }^{3} \mathrm{H}\right.$ ]pirenzepine binding and levels of mRNA (Supplementary Table 4). There were no correlations between any levels of $\mathrm{CpG}$ methylation and any demographic, brain collection of pharmacological variables (data not shown). Significantly, in the cohorts used for the study of miRNA there were correlations between levels of miR-16 and miR-107, but not any of the other measures in all diagnostic cohorts (Figure 3 and Supplementary Table 5).

CHRM1 reporter expression is modulated by intracellular miR-107. To further investigate the role of miR-107 in the regulation of CHRM1 we cloned a segment of its 3 UTR downstream of the Firefly luciferase reporter gene in PMIRREPORT and analysed reporter gene expression in HEK293 cells cotransfected with synthetic miR-107 or LNA modified antimiR-107 (miR-107 inhibitor). In each case, the Firefly
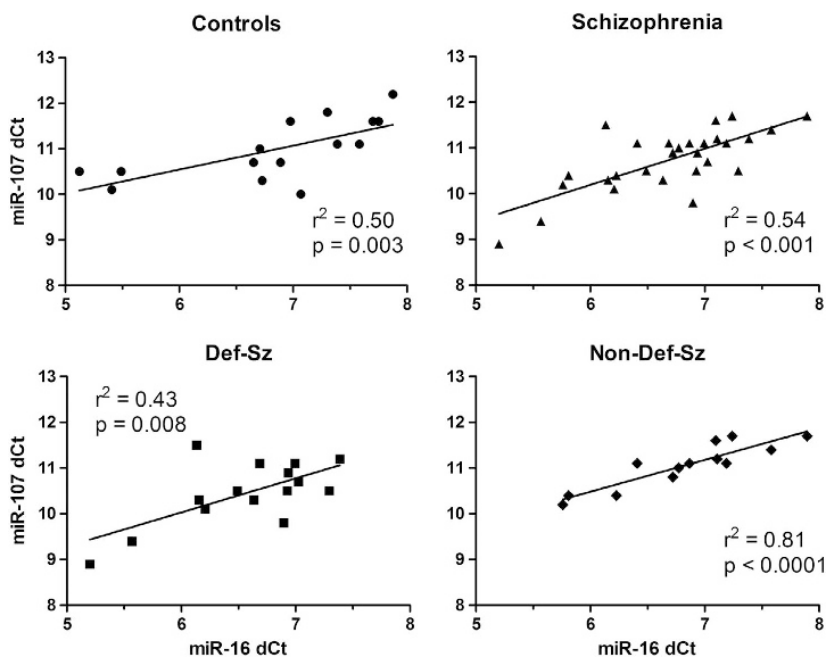

Figure 3 The relationship between levels of miR-16 and miR-107 in BA 9 from subjects with schizophrenia, Def-Sz, Non-Def-Sz and controls.

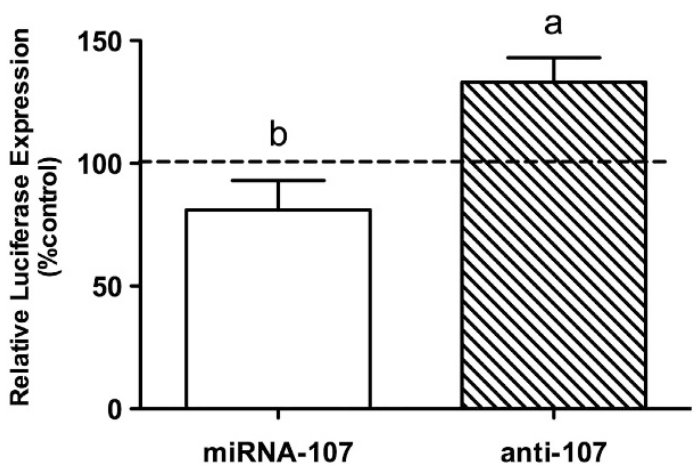

Figure 4 Effect (Mean \pm s.e.m.) of miR-107 and antimiR-107 on the activity of a CHRM1 reported gene construct. (a): $P<0.05$; (b): $P<0.005$. luciferase gene dosage was normalised by Renilla luciferase expressed from cotransfected $\mathrm{pRL}$-TK vector and compared with control transfections containing mutant versions of miR107 (and the corresponding LNA modified antimiRs). Following transfection, analysis of luciferase activity demonstrated that the addition of miR-107 was capable of reducing CHRM1 reporter gene expression by $20 \% \quad(P=0.004)$ (Figure 4). When the endogenous miR-107 was depleted or inhibited by its cognate anti-miRs, the reporter gene expression was increased by $62 \% \quad(P=0.03)$. These observations, showing gene silencing in the presence of miR-107 over-expression and a reduction of this effect when miR-107 is inhibited, demonstrate that this region of CHMR1 3' UTR is potently regulated by miR-107, at least in vitro.

Potential confounds. The only strong and significant relationships between experimental measures and potential numeric confounds was between $\left[{ }^{3} \mathrm{H}\right]$ pirenzepine binding and final recorded benzodiazepine drug dose $\left(r^{2}=0.68\right.$, $P<0.001)$ in people with $\mathrm{Sz}$ and between miR-107 and PMI $\left(r^{2}=0.47, P<0.01\right)$ in Non-Def-Sz (Supplementary Table 4). Effects of these relationships on the original analyses using an analysis of covariance showed that final recorded benzodiazepine drug dose as a covariate did not affect the variation of $\left[{ }^{3} \mathrm{H}\right]$ pirenzepine binding with diagnoses $(F=101$, d.f. $=12129, P<0.0001)$. When $P M I$ was included as a covariate in the analyse of $\mathrm{miR}-107$ it is notable that there is an increase in the variance associated with group $(F=4.94$, d.f. $=1,2,39, P=0.01$ ).

There was no effect of either gender $(P=0.63)$ or suicide $(P=0.17)$ on levels of $\left[{ }^{3} \mathrm{H}\right]$ pirenzepine binding in either people with $\mathrm{Sz}$ as a whole or their age/ sex matched controls. Levels of CHRM1 mRNA did not vary with gender or suicide in these cohorts $(P=0.41$ and $P=0.26$, respectively). In the cohorts used for the study of miRNA; levels of $\left[{ }^{3} \mathrm{H}\right]$ pirenzepine binding $(P=0.63), \quad C H R M 1$ mRNA $(P=0.87)$, miR-16 $(P=0.78)$ or miR-107 $(P=0.30)$ did not vary with gender when people with $\mathrm{Sz}$ were compared with controls. Similarly, levels of $\left[{ }^{3} \mathrm{H}\right]$ pirenzepine binding $(P=0.46), C H R M 1$ mRNA $(P=0.50)$, miR-16 $(P=0.26)$ and miR-107 $(P=0.13)$ did not vary with suicide.

This study explores the potential mechanisms that could contribute to widely replicated finding of reduced levels of cortical $\left[{ }^{3} \mathrm{H}\right.$ ]pirenzepine binding in people with $\mathrm{Sz}^{3,6,29-32}$ taking into account that, under the conditions used in most protocols, the radioligand will show $a \geqslant 80 \%$ selectivity for CHRM1. ${ }^{4,5}$ In addition, it explores whether changes in the status of markers of CHRM1 expression may differentiate people with Def-Sz, who have a $\sim 75 \%$ loss of cortical $\left[{ }^{3} \mathrm{H}\right]$ pirenzepine binding, ${ }^{3}$ from Non-Def-Sz. Consistent with previous findings ${ }^{6,33}$ we show a decrease in levels of cortical CHRM1 mRNA in Sz that is equivocal across Def-Sz and Non-Def-Sz. Our data also shows a decrease in levels of methylation of the putative promoter region of the CHRM1 gene in Sz. There was a significant decrease in the levels of methylation in four CpG sites in Non-Def-Sz whereas only three of these sites had significant decrease in levels of gene methylation in Def-Sz. This being noted, our overall analyses suggested differences in CHRM1 gene promoter methylation did not permit separation of the two groups. The presence of 
low levels of CHRM1 binding and mRNA has a symmetry but it is generally argued that increased gene methylation is associated with gene silencing and lower levels of mRNA. ${ }^{34}$ Thus, on the one hand our data is consistent with the suggestion that $\mathrm{Sz}$ is associated with a hypo-methylation state $^{35}$ but, on the other hand, our data also suggests such a state may not be associated with the expected increase in gene expression in the CNS of people with the disorder. Thus, our data leads us to postulate that the pathophysiology of $\mathrm{Sz}$ may interfere with the relationship between gene promoter methylation and levels of the gene expression.

Our study showed levels of miR-107, but not miR-16, were increased in the cortex from people with Def-Sz, but not NonDef-Sz. Significantly, from sequence homology, miR-107 was predicted to target $C H R M 1$ expression whereas miR-16 would not target CHRM1 mRNA. The notion that miR-107 targets CHRM1 expression is supported by our data showing changing miR-107 levels appropriately changes the activity of a reporter gene linked to levels of CHRM1 expression. Therefore our data suggests that $C H R M 1$ gene expression in people with Def-Sz is affected by the increased activity of at least one CHRM1 targeting miRNA and this unique pathophysiology could be causing the large decrease in cortical CHRM1 in these people. Overall, our data suggests that the changes in the regulation of CHRM1 expression is not a straightforward progression whereby changes in levels of mRNA predict changes in levels of protein; rather our data is in keeping with the concept of a derangement of the highly complex system of checks and balances regulating expression in the CNS. ${ }^{36}$

This study, for the first time, to our knowledge, offers an explanation as to an underlying mechanism that may be contributing to the marked decrease in cortical CHRM1 levels in Def-Sz. Our data suggests this mechanism involves increased levels of at least one miR, miR-107, in the cortex of people with Def-Sz and our in vitro data shows this increase in miR-107 would act to decrease CHRM1 expression and/or translation. Such a functional link is supported by our data showing a negative correlation between $\left[{ }^{3} \mathrm{H}\right]$ pirenzepine binding and levels of miR-107 $(r=-0.3671, P=0.001)$ in the cortex of people with Def-Sz that was not detectable in tissue from Non-Def-Sz and controls. We interpret these data as suggesting a stronger relationship between miR-107 and levels of CHRM1 in Def-Sz that would be expected if miR-107 was a significant factor in its pathophysiology. Importantly, levels of a miR-16, that does not target CHRM1 expression, is not altered in the cortex of Def-Sz and Non-Def-Sz showing the changes in miR-107 are not reflecting generalised changes in $\mathrm{miR}$ in the cortex of people with Def-Sz. Coalescing our data on CHRM1 binding, CHRM1 mRNA and miR-107 we now hypothesise that the marked decrease in CHRM1 levels in Def-Sz is due to the cumulative effects of decrease gene expression being amplified by the effects of increased $C H R M 1$ targeting miRNA activity.

Our data also adds to a growing body of data implicating gene methylation in the pathophysiology of Sz. ${ }^{37-41}$ However, our data differs in that it shows a decrease in CHRM1 expression (as measured by levels of mRNA) in the presence of decreased gene methylation, which would normally be expected to cause a decrease in gene silencing (that is, increased expression). ${ }^{34}$ Significantly, the expected inverse relationship between methylation and levels of gene expression has been reported in the face of decreased gene copy number in transgenic animals. ${ }^{42}$ Levels of gene methylation have long been known to be subject to feed-back loop regulation and it has been suggested that disruption of such processes could result in disease states. ${ }^{43}$ Thus, our data may reflect a disruptive feed-back loop involving methylation of CHRM1 as part of the pathophysiology of Sz because CNS gene methylation is a dynamic process that varies during life ${ }^{44}$ and the decreased methylation of the CHRM1 promoter region we report in Sz could be part of a feedback mechanism trying to turn on the expression of CHRM1 in an attempt to compensate for low levels of cortical mRNA and/or protein.

In conclusion, our study has identified changes in multiple markers (gene promoter methylation, levels of mRNA and protein) of CHRM1 gene expression in the dorsolateral prefrontal cortex from people with Sz. However, being able to analyse our data at the subsyndrome level has revealed an increase in a CHRM1 mRNA targeting miR (miR-107) only in the cortex of people with Def-Sz. We therefore hypothesise that this extra burden on CHRM1 gene expression is a significant contributor to the marked loss in cortical CHRM1 by which this subset of people is defined. Our data also supports that of others that have shown changes in levels of miRNA in the CNS of subjects with schizophrenia the suggest a role for miRNAs in the pathophysiology of $\mathrm{Sz}^{23}$ but extends this hypothesis to suggest that changes in miRNA levels may differentiate some subsets of people within the syndrome. However, we acknowledge that our sample size for this portion of our study is small and therefore our results must be regarded with caution until further data is obtained.

Our findings on the mechanisms underpinning changes in CHRM1 in Sz comes at a critical time given the development of CHRM1 allosteric modulates which, for the first time, open up the possibility of specifically targeting that receptor to bring about therapeutic benefits. ${ }^{45}$ Significantly, data on these new compounds support the hypothesis that they will be useful in reducing the symptoms of $S z^{46}$ but our data may indicate they may be useful in treating selective subgroups of people with the disorder. Hence, a high priority should be given to developing more selective CHRM1 ligands for neuroimaging, rather than the pan-muscarinic receptor ligand used to show decreased CHRMs in living people with $\mathrm{Sz},{ }^{47}$ to facilitate the testing of these new drugs in subjects who have a marked loss of CHRM1 compared to those who have not. Finally, our data strongly supports the argument that subdividing the syndrome of $\mathrm{Sz}$ will facilitate an improved understanding of its component disorders ${ }^{1}$ and that this understanding may lead to more personalised medicines with which to treat these component disorders. ${ }^{48}$

\section{Conflict of interest}

The authors declare no conflict of interest.

Acknowledgements. The authors are grateful for the excellent technical assistance of Mr Geoff Pavey. Tissues were received from the Victorian Brain Bank Network, which is supported by the Mental Health Research Institute, The Alfred, Victorian Forensic Institute of Medicine and The University of Melbourne and funded by Australia's National Health and Medical Research Council, Helen Macpherson 
Smith Trust, Parkinson's Victoria and Perpetual Philanthropic Services. Sources of support: BD is an NHMRC Senior Research Fellow (APP1002240). ES is an ARC Future Fellow (FT100100689). MSS is a recipient of a University of Melbourne International Research Scholarship. This research was principally funded by the NHMRC (Project Grant no. 509333 and 628669) but was also funded in part by the Victorian Government's Operational Infrastructure Support Programme, the Rebecca L Cooper Medical Research Foundation and the Wood's Family Research Program.

1. Tamminga CA. Accelerating new knowledge in schizophrenia. Am J Psychiatry 2008; 165 949-951.

2. Raedler TJ, Bymaster FP, Tandon R, Copolov D, Dean B. Towards a muscarinic hypothesis of schizophrenia. Mol Psychiatry 2007; 12: 232-246.

3. Scarr E, Cowie TF, Kanellakis S, Sundram S, Pantelis C, Dean B. Decreased cortical muscarinic receptors define a subgroup of subjects with schizophrenia. Mol Psychiatry 2009; 14: 1017-1023

4. Scarr E, Dean B. Muscarinic receptors: do they have a role in the pathology and treatment of schizophrenia? J Neurochem 2008; 107: 1188-1195.

5. Gibbons AS, Scarr E, Boer S, Money T, Jeon WJ, Felder C et al. Widespread decreases in cortical muscarinic receptors in a subset of people with schizophrenia. Int $J$ Neuropsychopharmacol; S1461145712000028 [pii]; doi:10.1017/S1461145712000028 (in press).

6. Dean B, McLeod M, Keriakous D, McKenzie J, Scarr E. Decreased muscarinic(1) receptors in the dorsolateral prefrontal cortex of subjects with schizophrenia. Mol Psychiatry 2002; 7 : 1083-1091.

7. Scarr E, Keriakous D, Crossland N, Dean B. No change in cortical muscarinic M2, M3 receptors or [35S]GTPgammaS binding in schizophrenia. Life Sci 2006; 78: 1231-1237.

8. Hill C, Keks N, Roberts S, Opeskin K, Dean B, Mackinnon A et al. Problem of diagnosis in postmortem brain studies of schizophrenia. Am J Psychiatry 1996; 153: 533-537.

9. Roberts SB, Hill CA, Dean B, Keks NA, Opeskin K, Copolov DL. Confirmation of the diagnosis of schizophrenia after death using DSM-IV: a Victorian experience. Aust N Z J Psychiatry 1998: 32: 73-76

10. Remington GJ. Antipsychotics (Neuroleptics). In B-Bjai JJ (ed) Handbook of Psychotropic Drugs. Hogrefe \& Huber: Seattle, Toronto, Gottingen, Bern, 1999, pp 55-84.

11. Dean B, Pavey G, Chai SY, Mendelsohn FAO. The localisation and quantification of molecular changes in the human brain using in situ radioligand binding and autoradiography. In Dean B, Kleinman JE, Hyde TM (ed) Using CNS tissue in psychiatric research: A practical guide. Harwood Academic Press: Amsterdam, 1999, pp 67-83.

12. Stan AD, Ghose S, Gao XM, Roberts RC, Lewis-Amezcua K, Hatanpaa KJ et al. Human postmortem tissue: what quality markers matter? Brain Res 2006; 1123: 1-11.

13. Kingsbury AE, Foster OJ, Nisbet AP, Cairns N, Bray L, Eve DJ et al. Tissue pH as an indicator of mRNA preservation in human post-mortem brain. Brain Res Mol Brain Res 1995; 28: 311-318.

14. Ollikainen M, Smith KR, Joo EJ, Ng HK, Andronikos R, Novakovic B et al. DNA methylation analysis of multiple tissues from newborn twins reveals both genetic and intrauterine components to variation in the human neonatal epigenome. Hum Mol Genet 2010; 19: 4176-4188.

15. Ewing B, Green P. Base-calling of automated sequencer traces using phred. II. Error probabilities. Genome Res 1998; 8: 186-194.

16. Fraga MF, Esteller M. DNA methylation: a profile of methods and applications. Biotechniques 2002; 33: 632, 634, 636-649.

17. Scarr E, Sundram $S$, Keriakous D, Dean B. Altered hippocampal muscarinic $m 4$, but not m1, receptor expression from subjects with schizophrenia. Biol Psychiatry 2007; 61 $1161-1170$.

18. Loiacono RE, Gundlach AL. In situ hybridisation histochemistry: application to human brain tissue. In Dean B, Hyde TM, Kleinman JE (ed) Using CNS Tissue in Psychiatric Research: A Practical Guide. Harwood Academic Press: Sydney, 1999, pp 85-106.

19. Dean B, Pavey G, Opeskin K. [3H]raclopride binding to brain tissue from subjects with schizophrenia: methodological aspects. Neuropharmacology 1997; 36: 779-786.

20. Megraw M, Sethupathy P, Corda B, Hatzigeorgiou AG. miRGen: a database for the study of animal microRNA genomic organization and function. Nucleic Acids Res 2007; 35 D149-D155.

21. Udawela M, Scarr E, Hannan AJ, Thomas EA, Dean B. Phospholipase $C$ beta 1 expression in the dorsolateral prefrontal cortex from patients with schizophrenia at different stages of illness. Aust N Z J Psychiatry 2011; 45: 140-147.

22. Santarelli DM, Beveridge NJ, Tooney PA, Cairns MJ. Upregulation of dicer and microRNA expression in the dorsolateral prefrontal cortex Brodmann area 46 in schizophrenia. Biol Psychiatry 2011; 69: 180-187.

23. Beveridge NJ, Gardiner E, Carroll AP, Tooney PA, Cairns MJ. Schizophrenia is associated with an increase in cortical microRNA biogenesis. Mol Psychiatry 2010; 15: 1176-1189.

24. Beveridge NJ, Tooney PA, Carroll AP, Tran N, Cairns MJ. Down-regulation of miR-17 family expression in response to retinoic acid induced neuronal differentiation. Cell Signal 2009; 21: $1837-1845$

25. D'Agostino RB, Belanger A, D'Agostino RB Jnr. A suggestion for using powerful and informative tests of normality. Am Stat 1990; 44: 316-321.

26. Cook RD, Weisberg S. Applied Regression Including Computing and Graphics. Wiley: Hoboken, 2011.

27. Dean B, Boer S, Gibbons A, Money T, Scarr E. Recent advances in postmortem pathology and neurochemistry in schizophrenia. Curr Opin Psychiatry 2009; 22: 154-160.

28. Falkai P, Wobrock T, Lieberman J, Glenthoj B, Gattaz WF, Moller HJ. World Federation of Societies of Biological Psychiatry (WFSBP) guidelines for biological treatment of schizophrenia, Part 1: acute treatment of schizophrenia. World J Biol Psychiatry 2005; 6: 132-191

29. Crook JM, Tomaskovic-Crook E, Copolov DL, Dean B. Low muscarinic receptor binding in prefrontal cortex from subjects with schizophrenia: a study of Brodmann's areas 8, 9, 10, and 46 and the effects of neuroleptic drug treatment. Am J Psychiatry 2001; 158: 918-925.

30. Zavitsanou K, Katsifis A, Filomena M, Xu-Feng H. Investigation of M1/M4 muscarinic receptors in the anterior cingulate cortex in schizophrenia, bipolar disorder, and major depression disorder. Neuropsychopharmacology 2004; 29: 619-625.

31. Deng C, Huang XF. Decreased density of muscarinic receptors in the superior temporal gyrus in schizophrenia. J Neurosci Res 2005; 81: 883-890.

32. Newell KA, Zavitsanou K, Jew SK, Huang XF. Alterations of muscarinic and GABA receptor binding in the posterior cingulate cortex in schizophrenia. Prog Neuropsychopharmacol Biol Psychiatry 2007; 31: 225-233.

33. Mancama D, Arranz MJ, Landau S, Kerwin R. Reduced expression of the muscarinic 1 receptor cortical subtype in schizophrenia. Am J Med Genet B Neuropsychiatr Genet 2003; 119: 2-6.

34. Turker MS. Gene silencing in mammalian cells and the spread of DNA methylation. Oncogene 2002; 21: 5388-5393.

35. Melas PA, Rogdaki M, Osby U, Schalling M, Lavebratt C, Ekstrom TJ. Epigenetic aberrations in leukocytes of patients with schizophrenia: association of global DNA methylation with antipsychotic drug treatment and disease onset. FASEB J 2012; 26: 2712-2718.

36. Qureshi IA, Mehler MF. The emerging role of epigenetics in stroke: II. RNA regulatory circuitry. Arch Neurol 2010; 67: 1435-1441.

37. Huang HS, Akbarian S. GAD1 mRNA expression and DNA methylation in prefrontal cortex of subjects with schizophrenia. PLOS ONE 2007; 2: e809.

38. Iwamoto K, Bundo M, Yamada K, Takao H, Iwayama-Shigeno Y, Yoshikawa T et al. DNA methylation status of SOX10 correlates with its downregulation and oligodendrocyte dysfunction in schizophrenia. J Neurosci 2005; 25: 5376-5381.

39. Grayson DR, Chen Y, Costa E, Dong E, Guidotti A, Kundakovic M et al. The human reelin gene: transcription factors $(+)$, repressors $(-)$ and the methylation switch $(+/-)$ in schizophrenia. Pharmacol Ther 2006; 111: 272-286.

40. Veldic M, Caruncho HJ, Liu WS, Davis J, Satta R, Grayson DR et al. DNA-methyltransferase 1 mRNA is selectively overexpressed in telencephalic GABAergic interneurons of schizophrenia brains. Proc Natl Acad Sci USA 2004; 101: 348-353.

41. Abdolmaleky HM, Yaqubi S, Papageorgis P, Lambert AW, Ozturk S, Sivaraman V et al. Epigenetic dysregulation of HTR2A in the brain of patients with schizophrenia and bipolar disorder. Schizophr Res 2011; 129: 183-190.

42. Garrick D, Fiering S, Martin DI, Whitelaw E. Repeat-induced gene silencing in mammals. Nat Genet 1998; 18: 56-59.

43. Jaenisch R, Bird A. Epigenetic regulation of gene expression: how the genome integrates intrinsic and environmental signals. Nat Genet 2003; 33(Suppl): 245-254.

44. Puckett RE, Lubin FD. Epigenetic mechanisms in experience-driven memory formation and behavior. Epigenomics 2011; 3: 649-664.

45. Conn PJ, Christopoulos A, Lindsley CW. Allosteric modulators of GPCRs: a novel approach for the treatment of CNS disorders. Nat Rev Drug Discov 2009; 8: 41-54.

46. Jones CK, Brady AE, Davis AA, Xiang Z, Bubser M, Tantawy MN et al. Novel selective allosteric activator of the M1 muscarinic acetylcholine receptor regulates amyloid processing and produces antipsychotic-like activity in rats. J Neurosci 2008; 28 : 10422-10433.

47. Raedler TJ, Knable MB, Jones DW, Urbina RA, Gorey JG, Lee KS et al. In vivo determination of muscarinic acetylcholine receptor availability in schizophrenia. Am J Psychiatry 2003; 160: 118-127.

48. Insel TR. Disruptive insights in psychiatry: transforming a clinical discipline. J Clin Invest 2009; 119: 700-705.

ब.

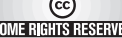

Translational Psychiatry is an open-access journal published by Nature Publishing Group. This work is licensed under the Creative Commons Attribution-NonCommercial-No Derivative Works 3.0 Unported License. To view a copy of this license, visit http://creativecommons.org/licenses/by-nc-nd/3.0/ 